\title{
A comparison between nuclear dismantling during plant and animal programmed cell death
}

\author{
Fernando Domínguez and Francisco Javier Cejudo
}

Instituto de Bioquímica Vegetal y Fotosíntesis, Universidad de Sevilla and CSIC, Avda Américo Vespucio 49, 41092-Sevilla, Spain

Correspondence to: Francisco Javier Cejudo; Instituto de Bioquímica Vegetal y Fotosíntesis, Universidad de Sevilla and CSIC, Avda Américo Vespucio 49, 41092Sevilla, Spain; E-mail: fjcejudo@us.es

Key words: apoptosis, plant, programmed cell death, nuclease, nucleus dismantling 


\begin{abstract}
Programmed cell death (PCD) is a process of organized destruction of cells, essential for the development and maintenance of cellular homeostasis of multicellular organisms. Cells undergoing PCD begin a degenerative process in response to internal or external signals, whereby the nucleus becomes one of the targets. The process of nucleus dismantling includes events affecting the nuclear envelope, such as formation of lobes at the nuclear surface, selective proteolysis of nucleoporins and nuclear pore complex clustering. In addition, chromatin condensation increases in coordination with DNA fragmentation. These processes have been largely studied in animals, but remain poorly understood in plants. The overall process of cell death has different morphological and biochemical features in plants and animals. However, recent advances suggest that nuclear dismantling in plant cells progresses with morphological and biochemical characteristics similar to those in apoptotic animal cells. In this review, we summarize nucleus dismantling in plant PCD, focusing on the similarities and differences with their animal counterparts.
\end{abstract}




\section{Contents}

1. Introduction

2. An overview of nuclear dismantling in animal cells

3. Similarities in nuclear dismantling in animal and plant cells undergoing PCD

3.1. Signalling that commits nuclear dismantling in plants

3.2. Sequence of events of plant nuclear dismantling during PCD

3.3. Nuclear dismantling is connected with cytoplasmic events during plant PCD

4. Is nuclear dismantling an autophagic process?

5. Concluding remarks and future perspectives

Acknowledgements

References 


\section{Introduction}

Programmed cell death (PCD) is a process of organized destruction of cells that maintains cellular homeostasis and is essential for the successful development of multicellular organisms [1]. PCD is an important process in plants, as in other multicellular organisms, not only for development [2], but also as a mechanism of immunity against pathogen attack [3]. Plant developmental cell death affects to determined cell types at precise stages of development and is characterized by the rupture of the vacuolar tonoplast and subsequent release of hydrolases, which degrade the cellular content and, in some cases, the cell wall. Cell death in biotic stress depends of the type of pathogens, but free radicals seem to exert an important function [4].

The plant cell has peculiar characteristics, most notably the presence of the cell wall and vacuoles, which suggest that the process of PCD takes place with different morphological features from apoptosis of animal cells. Indeed, most of the morphological features of mammalian apoptosis are not found in plant cells undergoing PCD and, thus, it has been proposed that cell death in plants does not take place by the process of apoptosis; however, the degree of conservation of plant and animal cell death programmes is at the moment a matter of debate [4]. The notion that plants seem to have evolved different mechanisms for PCD is reinforced by the biochemical analyses of the components involved in the execution of cell death in different plant systems. Despite intense search, caspases have not been found in plants whereas they are the most characteristic proteases executing apoptosis in animal cells. Still, there are caspase-like proteases involved in plant PCD [5]. Analyses carried out in different plant systems have identified several types of proteases involved in cell death, which include metacaspases [6], subtilisin-like proteases [7], and vacuolar processing enzymes [8]. Therefore, both morphological and biochemical characteristics suggest that plants and animals have developed different strategies that perform cell death.

The study of PCD has been a major field of research in different animal model systems not only because it is an essential process to understand development, but also because deregulation of PCD causes alterations of the cellular homeostasis, which have important implications associated with diseases, including cancer or neurodegenerative processes [9]. At the morphological level, animal PCD takes place by two wellestablished mechanisms, apoptosis and necrosis [10]. Apoptosis is characterized by active plasma membrane blebbing and cell fragmentation, forming the so-called 
apoptotic bodies, which are eventually engulfed by phagocytes. In parallel, the nucleus undergoes dramatic modifications, which include disorganization of the nuclear envelope, chromatin condensation and internucleosomal degradation of DNA in fragments of 180-200 bp and multimers of it, forming a characteristic ladder, which constitutes a hallmark of apoptosis [11]. In contrast, necrosis is characterised by the rupture of the plasma membrane and the consequent degradation of intracellular contents. Necrosis lacks the morphological features of apoptosis, notably the formation of apoptotic bodies. Increasing attention has been devoted to autophagy, a process of cell self-digestion in which cellular components are engulfed in vesicles, called autophagosomes, prior to their degradation by lysosomes. It is well-established that autophagy has pro-death functions, but this process may also have pro-survival functions, and the relationship of autophagy with apoptosis is still the subject of intense debate [12].

The nucleus is the major target of the cell degradation machinery at the onset of PCD. In apoptotic animal cells, the nucleus undergoes a massive reorganization, which includes the condensation of chromatin and internucleosomal fragmentation of DNA [11]. Condensation of chromatin has also been described in different plant systems. This is the case of nuclei from nucellar cells undergoing PCD during wheat grain development, the chromatin of which condenses in a morphologically similar manner as occurs in apoptotic animal cells [13]. Similarly, DNA laddering is also a hallmark of PCD in different plant systems such as pea carpel senescence [14]. However, it should be noted that both chromatin condensation and DNA laddering are not as consistent PCD markers in plants as they are in animal cells. Therefore, despite the clear morphological and biochemical differences of PCD in plants and animals, the phase of nuclear dismantling seems to have similarities in both types of cells. Indeed, factors involved in nuclear dismantling from plant cells are able to induce apoptotic morphology and DNA fragmentation in human cells [15], which suggests the possibility of common mechanisms in PCD of cells from both kingdoms, at least at the stage of nucleus dismantling.

In this review, we will summarize the current knowledge of the process of nuclear dismantling during PCD in plant systems, discussing the similarities and differences with the process in animal systems.

\section{An overview of nuclear dismantling in animal cells}


The content of the nucleus, the nucleoplasm, is separated from the cytoplasm by a complex membranous nuclear envelope formed by outer and inner nuclear membranes, which define the perinuclear space. The nuclear envelope is penetrated by nuclear pore complexes that mediate the nucleo-cytoplasmic interchange in both directions [16]. The nuclear matrix at the internal side of the nuclear envelope acts as a skeleton defining nuclear size and shape. Several nuclear membrane proteins localized to the inner side of the nuclear envelope provide binding sites for chromatin and nuclear matrix in animals and plants. In animal cells the nuclear matrix is composed of lamins and lamin-associated proteins [17], nuclei of plant cells lack this structure, with scaffold and structural support exerted by coiled proteins [18].

Morphological and biochemical analyses have led to considerable advances of our knowledge of nuclear dismantling during animal apoptosis [11]. The protein profile of the nucleus in cells undergoing apoptosis is modified by the appearance of transcription factors, protein kinases, proteases and DNases, among other proteins. These proteins are probably translocated from the cytoplasm, as schematically shown in Figure 1. One of the earliest events taking place during nuclear dismantling is the proteolysis of matrix attachment region-binding proteins, which anchor chromatin to the scaffold [19]. This limited cleavage may open nuclease sites on the chromatin structure allowing fragmentation of DNA, which facilitates the subsequent proteolysis of the bulk of nuclear matrix proteins (Figure 1). Several membrane proteins localized to the inner side of the nuclear envelope, such as lamin B receptor, lamin-associated polypeptide $2 \alpha$ and nucleoporin Nup 153, are connected to chromatin. The cleavage of these proteins promotes the detachment of chromatin from the nuclear envelope, which results in nuclear pore clustering. Finally, it has been speculated that cleavage of components of the nuclear pore complex and the nuclear transport machinery may stimulate an increase in nuclear pore permeability, facilitating protein translocation from the cytoplasm into the nucleus [19].

Post-translational modifications of nuclear proteins seem to have an important function in nuclear dismantling during apoptosis. Histones H2, H3 and H4, lamins and HMGA1a protein are hyperphosphorylated, whereas histone $\mathrm{H} 1$ is dephosphorylated just before DNA fragmentation [19]. It is worth comparing nuclear envelope disassembly during mitosis and apoptosis because disorganization of this structure may share common mechanisms in both processes. Nup98, a peripheral nucleoporin 
localized on both sides of the nuclear envelope, contains 13 phosphorylation sites that are successively phosphorylated by mitotic kinases, driving nuclear pore complex disassembly and nuclear envelope permeabilization during mitosis [20]. Hyperphosphorylation of Nup98 and other nucleoporins also seems to have a key regulatory role in apoptosis. The post-translational modifications of nuclear proteins are presumably important for three events: chromatin condensation, accessibility of nucleases to DNA and the breakdown of the lamina.

\section{Similarities in nuclear dismantling in animal and plant cells undergoing PCD}

As mentioned above, animal cell nuclei undergo very characteristic morphological changes during apoptosis. The nucleus becomes fragmented and the fragments move to apoptotic bodies, which are subsequently phagocytosed by macrophages, parenchymal or neoplastic cells and degraded by phagolysosomes [21]. Therefore, the nucleus of apoptotic animal cells becomes degraded inside another cell. In contrast, in plant cells undergoing PCD, the nucleus is not degraded in another cell, which is a clear difference of nuclear dismantling between plant and animal cell death.

Despite these differences, nuclear extracts of etopoxide-induced apoptotic human cells triggered apoptotic morphology of plant cell nuclei [15]. In the same way nuclear extracts from wheat nucellar cells undergoing PCD induce apoptotic morphology and DNA fragmentation in nuclei from human cells (Figure 2). These results suggest the existence of common mechanisms in the machinery of nuclear dismantling in animal and plant cells.

CAD (Caspase Activated DNase) is the first nuclease in apoptotic animal cells responsible for the characteristic internucleosomal degradation of DNA [22]. CAD is maintained in an inactive status by the formation of a complex with its inhibitor [23]. The cleavage of the inhibitor in apoptotic cells releases active CAD, thus allowing the subsequent degradation of DNA [24]. The finding that nuclear extracts from plant cells undergoing PCD can trigger apoptotic morphology and DNA fragmentation in nuclei from human cells prompted us to search for common elements of nuclear dismantling in animal and plant cells. No candidate genes have been identified so far encoding putative CAD or its inhibitor in plants. Apoptosis-inducing factors extracted from nuclei of plant cells undergoing PCD were not inhibited by mammalian CAD inhibitors (Figure 2B). $\mathrm{Zn}^{2+}$ and EDTA, inhibitors of PCD-related $\mathrm{Ca}^{2+}$ and/or $\mathrm{Mg}^{2+}$-dependent endonucleases 
[15], inhibited the induction of animal apoptosis (Figure 2B). These results suggest that a CAD type nuclease is not responsible for DNA fragmentation in plant cell nuclei undergoing PCD. Therefore nuclear dismantling in plants seems to involve different factors from those in animals. Search for plant homologues of the components of animal cell apoptosis has been carried out by bioinformatic approaches based on sequence similarity, which have not given positive results. The identification of possible shared genes will require searches based on structure and function rather than sequence.

\subsection{Signalling that commits nuclear dismantling in plants}

The process of PCD leading to nuclear dismantling can be schematically subdivided into three major phases: signalling, execution and nuclear dismantling itself. The signalling and execution phases of PCD are beyond the scope of this review. Still, some recent advances in model plant systems, which suggest functional conservation of key events regulating PCD, are here discussed. The rice transcription factor OsNAC4 is translocated into the nucleus in a phosphorylation-dependent manner in pathogentriggered hypersensitive responses. It activates a $\mathrm{Ca}^{2+}$-dependent nuclease, which promotes nuclear DNA fragmentation [25]. The metacaspase mcII-Pa is translocated from the cytoplasm to the nucleus during Norway spruce (Picea abies) embryogenesis and facilitates cell death by cleaving a phylogenetically conserved nuclease $[26,27]$. A mitogen-activated protein kinase appears as a signalling factor during early PCD in Papaver rhoeas pollen, stimulating DNA fragmentation [28].

The pathway of PCD culminates in the process of nuclear dismantling in both plant and animal cells. As discussed above, the nuclei of plant and animal cells have very similar structures and, despite the differences, undergo similar modifications, the most relevant being chromatin condensation and DNA fragmentation, in dying cells. Thus the question arises whether the key events of nuclear dismantling are similar in both types of cells. The most relevant events taking place during nuclear dismantling in animal and plant PCD are summarized in Table 1. The first event is the translocation of different factors and enzymes to the nucleus that initiate the process. In apoptotic animal cells the most relevant process that takes place at this stage is the translocation of the CAD complex with its inhibitor [23, 24]. The relevant event in plants at this stage is the translocation of transcription factors, such as OsNAC4 [25], and the metacaspase mcII- 
$\mathrm{Pa}$ [26]. Then, the maturation of apoptogenic factors, or inactivation of their inhibitors, occurs. The relevant activity illustrating this stage is the cleavage of the inhibitor of CAD by caspase-3 in animal cells undergoing apoptosis [24], and the cleavage of the Tudor Staphylococcal Nuclease (TSN) by metacaspase mcII-Pa [27] in plant PCD. The disruption of the nucleo-cytoplasmic barrier, facilitated by limited digestion of some nucleoporins of the nuclear pore complex, which alters the permeability through the pore of an exclusion size of ca. $30 \mathrm{kDa}$ to more than $70 \mathrm{kDa}$, has been documented in apoptotic animal cells [29] but, to our knowledge, not in plant cells undergoing PCD. Similarly, the protection from cleavage of other nucleoporins until the end of the death process has been documented only in apoptotic animal cells [30], but not in plant cells.

A series of events follows with the cleavage of DNA into different conformations (chromatin loop DNA, oligonucleosomal DNA or naked DNA) catalysed by different nucleases. Examples of these nucleases have been reported both in animal and plant PCD (Table 1). Finally, nuclear pore complex clustering, which may facilitate nuclear fragmentation and chromatin release to the cytoplasm, as well as engulfment of nuclear fragments, have also been reported in both types of cells [13, 19, 32, 36].

\subsection{Sequence of events of plant nuclear dismantling during PCD}

Two types of events can be distinguished in the process of nuclear dismantling; those affecting chromatin structure and DNA fragmentation, and those affecting the nuclear envelope. The most characteristic structural feature in cells undergoing PCD is that chromatin becomes increasingly condensed. This process begins at the inner side of the nuclear envelope, where discrete patches of condensed chromatin can be observed. In animal cell apoptosis the progression of chromatin condensation can be classified in three stages: stage I, or ring condensation, stage II, or necklace condensation, and stage III, or nuclear collapse/disassembly [37, 38], as schematized in Figure 3A. These stages are less clearly defined in plant cells, but the process of chromatin condensation progresses essentially in the same way and culminates in the formation of discrete domains of condensed chromatin $[15,26]$. Two types of chromatin condensation morphologies appear during petal senescence: a) fragments of condensed chromatin inside the nucleus and b) a large number of DNA spherical bodies, each enveloped by a 
membrane in the cytoplasm [39]. The second phenomenon seems to be due to nuclear fragmentation.

DNA fragmentation proceeds in a precise order, which is related to the progression of chromatin condensation (Figure 3B). At early stages of PCD, highmolecular-weight DNA cleavage produces fragments of 50-300 kb, corresponding to the size of the chromatin loops; later, the characteristic DNA ladder of multiples of ca. 180-200 bp arises as consequence of internucleosomal, low-molecular-weight DNA fragmentation. Finally, cleavage of single-stranded DNA completes the process of DNA degradation. Extensive analyses of these processes have allowed the identification of different nucleases involved in the three stages in apoptotic animal cells [11]. Different nucleolytic activities have been described to participate in plant PCD in a similar three level-DNA fragmentation, as summarized in Table 1. Chromatin loop endonucleolytic activities have been described in PCD-related developmental processes, such as embryogenesis [32]. Endonucleases promoting DNA internucleosomal fragmentation were found in cells undergoing PCD in response to abiotic stress, such as D-mannose treatment [33], and cells undergoing PCD in developing or germinating seeds [15, 34, 40]. The final single-stranded DNA cleavage and their corresponding nucleases appear in almost any plant PCD system, including senescence [41] or xylem vessel differentiation [35].

Morphological and biochemical evidence suggests that nuclear envelope and nuclear pore disassembly is a tightly regulated process in animal cell apoptosis. The disassembly of the nuclear pore complex during apoptosis is a sequential process, which might be divided into the three stages previously described in coordination with the progress of chromatin condensation and DNA fragmentation, as depicted in Figure 3C. Stage I is characterized by the limited proteolysis of specific nucleoporins of the central core complex framework, which affects the interaction with other nucleoporins that promote nucleo-cytoplasmic translocation [30]. The peripheral nucleoporin, RanBP2/Nup358, comprising the cytoplasmic fibrils, is also degraded. No other nucleoporin of the central core seems to be degraded at this early stage, in agreement with the fact that the morphology of the nuclear pore remains intact until the final stages of apoptosis. Stage II of nuclear pore complex disassembly is characterized by lamin B and LAP2 breakdown and the cleavage of peripheral nucleoporins. The cleaved nucleoporins include Tpr and Nup 153, which comprise the nuclear basket at the nucleoplasmic side of the nuclear envelope, and Nup 50, a protein that moves from this 
side to the interior of the core structure [42]. The cleavage of Nup 214 completes the degradation of the cytoplasmic fibrils on the cytoplasmic side of the nuclear envelope [29, 30]. Lamins, LAP2 and Nup 153 interact with chromatin and contribute to the anchorage of the nuclear pore complex to the nuclear envelope. Thus, their proteolysis allows the detachment of condensed chromatin and clustering of the nuclear pore complex [19]. Finally, stage III is characterized by the loss of turgor of the nucleus while the nuclear envelope softens, allowing nuclear fragmentation [29]. Potential cleavage sites have been described in Nup 62 [31], but this nucleoporin remains intact until later stages of apoptosis.

The plant nuclear pore complex resembles those in yeast and animal cells, and a lamina-like structure is seen on the inner side of the nuclear envelope [43]. The components of the nuclear pore complex in Arabidopsis thaliana have been identified and only seven animal nucleoporins seem to be absent in plants. The rest of the components show high homology with their counterparts in animal cells, and the threedimensional structure of the overall complex is highly similar [44]. However, the process of nuclear pore complex disassembly in plant cells undergoing PCD is still poorly understood. By analogy between plant and animal nuclear pore complex [45], it can be anticipated that Nup 93, Nup 96, Tpr/NUA, Nup 136, Nup 50, Nup 214 and proteins from the plant lamin-like structure might be potential substrates for plant proteases involved in PCD, but much work is still needed to confirm this possibility.

There is no strict correlation between cytoplasm degradation and nuclear dismantling in plant systems. Senescent leaf cells show a clear order of intracellular organelle disintegration. The earliest event of leaf senescence is chloroplast degeneration, while mitochondria and the nucleus seem to be intact until final stages. Vacuolar collapse at the end of senescence promotes the degeneration of the nucleus, characterized by DNA degradation and chromatin condensation among other symptoms [46]. A large set of genes, the so-called Senescence-Associated Genes (SAGs), encoding different types of hydrolytic enzymes and other factors, are upregulated during leaf senescence [47, 48]. The catabolic phase of senescence and the recycling of nutrients are tightly regulated processes that require the differential expression of a large set of genes, so that the nucleus is the last organelle to be removed. The finding of specific transcription factors involved in leaf senescence $[49,50]$ constitute an exciting area of research at the moment. 
The nucleus is also the last organelle to be dismantled during tracheary element differentiation. Vacuolar collapse occurs at the end of the differentiation process, releasing hydrolytic enzymes that promote the destruction of the nucleus [51]. Paradoxically, the DNA of secondary xylem cells (xylary fibres) is gradually degraded and nuclei disintegrate long before the cells die, suggesting controlled dismantling prior to cell death in contrast to tracheary elements, where a post-mortem DNA degradation takes place after vacuole collapse [52].

Nuclear dismantling during leave senescence and tracheary element differentiation does not occur according to the three phases described in Figure 3. Therefore, condensed chromatin and DNA laddering may not always be detected in tissues where the cell death is markedly asynchronous, such as senescing petals [53], or where the DNA is rapidly degraded after vacuolar collapse, such as in differentiating xylem cells [54].

\subsection{Nuclear dismantling is connected with cytoplasmic events during plant PCD}

The key events in the cytoplasm and nucleus of animal cells undergoing apoptosis have been well described. Knowledge about the connection between cytoplasm and nucleus in plant cells undergoing PCD is still scarce. There has been an increasing interest in identifying nuclear-localized factors involved in plant PCD. Different organelles provide crucial factors, which directly or indirectly affect nuclear dismantling in plant cells, as summarized in Table 2. This is the case with the endoplasmic reticulum, which accumulates and releases cysteine proteases [55] as well as nucleases involved in PCD [41], and the Golgi, which participates in the trafficking of proteases to the vacuoles [56]. The vacuole plays an important role in plant cell death. The vacuolar processing enzyme is processed and several proteolytic and nucleolytic enzymes accumulate, and are liberated upon tonoplast disruption $[8,57]$. Endonucleases produced and released from mitochondria participate in nuclear DNA degradation, similar to animal cells [58]. Cytochrome $\mathrm{c}$ and other apoptogenic factors are apparently also released from the mitochondria and may play important roles after its possible translocation/diffusion to the nucleus [58]. Chloroplast produces reactive oxygen species during cellular stress, affecting and regulating PCD [59]. Finally, reorganization of the cytoskeleton network is also important for autophagosome formation and dynamics, driving movements for cell content engulfment [60]. 


\section{Is nuclear dismantling an autophagic process?}

Autophagy is a process of cell degeneration in animals that, besides apoptosis, may participate in the death process. Autophagy is characterized by the presence of double-membrane autophagosomes within the dying cells that remove the cell remnants [12]. Nucleophagy, a selective process of autophagy, was first described as a novel way in which nonessential or damaged components of yeast nuclei are recycled by the generation of a specific nucleus-vacuole junction by which nuclear blebs are released into the vacuole lumen and degraded by soluble hydrolases [61]. Nucleophagy also occurs in mammalian cells [62] and fungi [63], where the entire nucleus is targeted. It appears that the core autophagy genes are needed for this nucleophagic process. There is no evidence for a specific nucleophagy process in plants, but the nucleus may undergo either a process of macroautophagy, in which portions are sequestered within doublemembrane autophagosome vesicles, or microautophagy, which occurs by direct invagination of the vacuole membrane [64].

'Autolytic cell death' was defined as the form of plant PCD in which lytic vacuoles play an important function [4]. Breakdown of the nucleus is often part of this autolytic process and the nucleus is degraded as cell dies. Nuclear degeneration starts prior to death in other cells in which remnants of condensed chromatin and nuclear pore complexes were observed within provacuoles or in the large central vacuole [32]. Similarly, heterochromatin aggregates devoid of the nuclear envelope were detected in highly-vacuolated-nucellar cells of developing wheat grains undergoing PCD [13].

\section{Concluding remarks and future perspectives}

A major aspect to be addressed in plant nucleus dismantling is the identification of factors involved in chromatin condensation. Chromatin condensation in apoptotic animal cells is controlled by at least two types of factors, ring-condensation factors and ATP-dependent condensation factors. The role of acinus, a caspase-3 activated protein involved in chromatin condensation has also been established $[65,66]$. However, the molecular basis of chromatin condensation (Figure 3A) is still poorly understood in plant PCD. 
A variety of endonucleolytic activities controlling DNA fragmentation have been characterized in plants (Figure 3B). Some are involved in PCD-related processes, but their molecular characterization is mostly lacking. The search for plant homologues, and factors regulating this activity in PCD, are major challenges. Approaches based on sequence similarity searches have failed so far to identify these genes. Hopefully, structure or function-based searches may be the way to solve this problem.

More research is also required to establish the function of various proteolytic activities in cell death. Approaches to identify plant caspases or caspase-like activities in plant cells undergoing PCD led to the identification of metacaspases, vacuolar processing enzymes, phytaspases and other proteolytic enzymes. More recently, an additional approach has been suggested, based on the identification of plant protease substrates, which seem to have a higher degree of conservation than the proteases [67].

Finally, a very relevant aspect of nuclear dismantling has to do with the disassembly of the nuclear envelope and the behaviour of the nuclear pore complex. Different proteins have been identified that might serve as markers of nuclear envelope dynamics [45]. It is possible to decipher the hierarchical cascade of events during nuclear envelope dismantling in plant PCD (Figure 3C). Moreover, the availability of mutants deficient in components of the nuclear envelope and nuclear pore complex may improve our knowledge about the involvement of these structures in nucleus dismantling. The disruption in the lamin-like proteins LINC 1 and 2 produce alterations of nuclear morphology [68]. Future progress in understanding nuclear structures and dynamics will assist research on nuclear dismantling.

\section{Acknowledgements}

Work in our lab is financed by ERDF-co-financed grants from Ministry of Science and Innovation (BIO2010-15430) and the Junta de Andalucía (BIO-182 and CVI-5919).

\section{References}

[1] N.K. Danial, S.J. Korsmeyer, Cell death: critical control points. Cell 116 (2004) 205-219.

[2] H. Kuriyama, H. Fukuda, Developmental programmed cell death in plants. Curr. Opin. Plant Biol. 5 (2002) 568-573.

[3] D. Hofius, et al., Autophagic components contribute to hypersensitive cell death in Arabidopsis. Cell 137 (2009) 773-783. 
[4] W.G. van Doorn, Classes of programmed cell death in plants, compared with those in animals. J. Exp. Bot. 62 (2011) 4749-4761.

[5] O. Del Pozo, E. Lam, Caspases and programmed cell death in the hypersensitive response of plants to pathogens. Curr. Biol. 8 (1998) 1129-1132.

[6] L.Tsiatsiani, et al., Metacaspases. Cell Death Diff. 18 (2011) 1279-1288.

[7] A.B. Vartapetian, A.L. Tuzhikov, N.V. Chichkova, M. Taliansky, T.J. Wolpert, A plant alternative to animal caspases: subtisilin-like proteases. Cell Death Diff. 18 (2011) 1289-1297.

[8] I. Hara-Nishimura, N. Hatsugai, M. Kuroyanagi, S. Nakaume, M. Nishimura, Vacuolar processing enzyme: an executor of plant cell death. Curr. Op. Plant Biol. 8 (2005) 404-408.

[9] Y. Fuchs, H. Steller, Programmed cell death in animal development and disease. Cell 147 (2011) 742-758.

[10] M. Leist, M. Jäätelä, Four deaths and a funeral: from caspases to alternative mechanisms. Nat. Rev. Mol. Cell Biol. 3 (2001) 589-598.

[11] K. Samejima, W.C. Earnshaw, Trashing the genome: The role of nucleases during apoptosis. Nat. Rev. Mol. Cell Biol. 6 (2005) 677-688.

[12] J.M. Gump, A. Thorburn, Autophagy and apoptosis: what is the connection? Trends Cell Biol. 21 (2011) 387-392.

[13] F. Domínguez, J. Moreno, F.J. Cejudo, The nucellus degenerates by a process of programmed cell death during the early stages of wheat grain development. Planta 213 (2001) 352-360.

[14] D. Orzáez, A. Granell, DNA fragmentation is regulated by ethylene during carpel senescence in Pisum sativum. Plant J. 11 (1997) 137-144.

[15] F. Domínguez, F.J. Cejudo, Identification of a nuclear-localized nuclease from wheat cells undergoing programmed cell death that is able to trigger DNA fragmentation and apoptotic morphology on nuclei from human cells. Biochem. J. 307 (2006) 529-536.

[16] M.A. D'Angelo, M.W. Hetzer, Structure, dynamics and function of nuclear pore complexes. Trends Cell Biol. 18 (2008) 456-466.

[17] Y. Gruenbaum, A. Margalit, R.D. Goldman, D.K. Shumaker, K.L. Wilson, The nuclear lamina comes of age. Nat. Rev. Mol. Cell Biol. 6 (2005) 21-31.

[18] F. Gindullis, A. Rose, S. Patel, I. Meier, Four signature motifs define the first class of structurally related large coiled-coil proteins in plants. BMC Genomics 3 (2002) 9.

[19] A.M. Martelli, et al., Nuclear apoptotic changes: an overview. J. Cell. Biochem. 82 (2001) 634-646.

[20] E. Laurell, et al., Phosphorylation of Nup 98 by multiple kinases is crucial for NPC disassembly during mitotic entry. Cell 114 (2011) 539-550.

[21] S. Elmore, Apoptosis: a review of programmed cell death. Toxicol Pathol. 35 (2007) 495-516.

[22] X. Liu, et al., The 40-kDa subunit of DNA fragmentation factor induces DNA fragmentation and chromatin condensation during apoptosis. Proc. Natl. Acad. Sci. USA 95 (1998) 8461-8466.

[23] M. Enari, et al., A caspase-activated DNase that degrades DNA during apoptosis, and its inhibitor ICAD. Nature 391 (1998) 43-50.

[24] H. Sakahira, M. Enari, S. Nagata, Cleavage of CAD inhibitor in CAD activation and DNA degradation during apoptosis. Nature 391 (1998) 96-99.

[25] T. Kaneda, et al., The transcription factor OsNAC4 is a key positive regulator of plant hypersensitive cell death. EMBO J. 28 (2009) 926-936. 
[26] P.V. Bozhkov, et al., Cysteine protease mcII-Pa executes programmed cell death during plant embryogenesis. Proc. Natl. Acad. Sci. USA 102 (2005) 14463-14468.

[27] J.F. Sundtröm, et al., Tudor staphylococcal nuclease is an evolutionarily conserved component of the programmed cell death degradome. Nat. Cell Biol. 11 (2009) 1347-1354.

[28] S. Li, Y. Samaj, V.E. Franklin-Tong, A Mitogen-Activated Protein Kinase signals to programmed cell death induced by self-incompatibility in Papaver pollen. Plant Physiol. 145 (2007) 235-245.

[29] A. Kramer, et al., Apoptosis leads to a degradation of vital components of active nuclear transport and a dissociation of the nuclear lamina. Proc. Natl. Acad. Sci. USA 105 (2008) 11236-11241.

[30] M. Patre, et al., Caspases target only two architectural components within the core structure of the nuclear pore complex. J. Biol. Chem. 281 (2006) 1296-1304.

[31] F. Oberhammer et al., Apoptotic death in epithelial cells: cleavage of DNA to 300 and/or $50 \mathrm{~kb}$ fragments prior to or in the absence of internucleosomal fragmentation. EMBO J. 12 (1993) 3679-3684.

[32] L.H. Filonova, et al.Two waves of programmed cell death occur during formation and development of somatic embryos in the gymnosperm, Norway spruce. J. Cell Sci. 113 (2000) 4399-4411.

[33] J.C. Stein, G. Hansen, Mannose induces an endonuclease responsible for DNA laddering in plant cells. Plant Physiol. 121 (1999) 71-79.

[34] F. Domínguez, J. Moreno, F.J. Cejudo, A gibberellin-induced nuclease is localized in the nucleus of wheat aleurone cells undergoing programmed cell death. J. Biol. Chem. 279 (2004) 11530-11536.

[35] J. Ito, H. Fukuda, ZEN1 is a key enzyme in the early degradation of nuclear DNA during programmed cell death of tracheary elements. Plant Cell 14 (2002) 32013211.

[36] L.P. Erwig, P.M. Henson, Clearance of apoptotic cells by phagocytes. Cell Death Diff. 15 (2008) 243-250.

[37] M. Kihlmark, G. Imreh, E. Hallberg, Sequential degradation of proteins from the nuclear envelope during apoptosis. J. Cell Sci. 114 (2001) 3643-3653.

[38] S. Toné, et al., Three distinct stages of apoptotic nuclear condensation revealed by time-lapse imaging, biochemical and electron microscopy analysis of cell-free apoptosis. Exp. Cell Res. 313 (2007) 3635-3644.

[39] T. Yamada, K. Ichimura, W.G. Van Doorn, DNA degradation and nuclear degeneration during programmed cell death in Antirrhinum, Argyranthemum and Petunia. J. Exp. Bot. 57 (2006) 3543-3552.

[40] F. Domínguez, J. Moreno, F.J. Cejudo, The scutellum of germinated wheat grains undergoes programmed cell death: identification of an acidic nuclease involved in nucleus dismantling. J. Exp. Bot. 63 (2012) 5475-5485.

[41] S. Farage-Barhom, et al., Localization of the Arabidopsis senescence- and cell death-associated BFN1 nuclease: from the ER to fragmented nuclei. Mol. Plant 4 (2011) 1062-1073.

[42] E. Ferrando-May, et al., Caspases mediate nucleoporin cleavage, but not early redistribution of nuclear transport factors and modulation of nuclear permeability in apoptosis. Cell Death Diff. 8 (2001) 495-505.

[43] J. Fiserova, E. Kiseleva, M.W. Goldberg, Nuclear envelope and nuclear pore complex structure and organization in tobacco BY-2 cells. Plant J. 59 (2009) 243255. 
[44] K. Tamura, Y. Fukao, M. Iwamoto, T. Haragushi, I. Hara-Nishimura, Identification and characterization of nuclear pore complex components in Arabidopsis thaliana. Plant Cell 22 (2010) 4084-4097.

[45] J. Boruc, X. Zhou, I. Meier, Dynamics of the plant nuclear envelope and nuclear pore. Plant Physiol. 158 (2012) 78-86.

[46] P.O. Lim, H.J.Kim, H.G. Nam, Leaf senescence. Annu. Rev. Plant Biol. 58 (2007) 115-136.

[47] V. Buchanan-Wollaston et al., Comparative transcriptome analysis reveals significant differences in gene expression and signalling pathways between developmental and dark/starvation-induced senescence in Arabidopsis. Plant J. 42 (2005) 567-58.

[48] E. Breeze, et al., High-resolution temporal profiling of transcrips during Arabidopsis leaf senescence reveals a distinct chronology of processes and regulation. Plant Cell 23(2011) 873-894.

[49] S-D.Yang, P.J. Seo, H-K Yoog, C-M. Park, The Arabidopsis NAC Transcription factor VNI2 integrates abscisic acid signals into leaf senescence via the COR/RD. Plant Cell 23 (2011) 2155-2168.

[50] A. Wu, et al., Jungbrunnen1, a reactive oxygen species-responsive NAC transcription factor, regulates longevity in Arabidopsis. Plant Cell 24 (2012) 482506.

[51] S. Turner, P. Gallois, D. Brown, Tracheary element differentiation. Annu. Rev. Plant Biol. 58 (2007) 407-433.

[52] C.L. Courtois-Moreau et al., A unique program for cell death in xylem fibers of Populus stem. Plant J. 58 (2009) 260-274.

[53] R. Battelli, et al., Changes in ultrastructure, protease and caspase-like activities during flower senescence in Lilium longiflorum. Plant Sci. 180 (2011) 716-725.

[54] B. Bollhöner, J. Prestele, H. Tuominen, Xylem cell death: emerging understanding of regulation and function, J. Exp. Bot. 63 (2012) 1081-1094.

[55] J.S. Greenwood, M. Helm, C. Gietl, Ricinosomes and endosperm transfer cell structure in programmed cell death of the nucellus during Ricinus seed development. Proc. Natl. Acad. Sci. USA 102 (2005) 2238-2243.

[56] C. Andeme-Ondzighi, D.A. Christopher, E.J. Cho, S-C. Chang, A. Staehelin, Arabidopsis protein disulfide isomerase-5 inhibits cysteine proteases during trafficking to vacuoles before programmed cell death of the endothelium in developing seeds. Plant Cell 20 (2008) 2205-2220.

[57] I. Hara-Nishimura, N. Hatsugai, The role of vacuole in plant cell death. Cell Death Diff. 18 (2011) 1298-1304.

[58] J. Balk, S.K. Chew, C.J. Leaver, P.F. McCabe, The intermembrane space of plant mitochondria contains a DNase activity that may be involved in programmed cell death. Plant J. 34 (2003) 573-583.

[59] S.M. Doyle, M. Diamond, P.F. McCabe, Chloroplast and reactive oxygen species involvement in apoptotic-like programmed cell death in Arabidopsis suspension cultures. J. Exp. Bot. 61 (2010) 473-482.

[60] A.P. Smertenko, P.V. Bozhkov, L.H. Filonova, S. von Arnold, P.J. Hussey, Reorganization of the cytoskeleton during developmental programmed cell death in Picea abies embryos. Plant J. 33 (2003) 813-824.

[61] P. Roberts, et al., Piecemeal microautophagy of nucleus in Saccharomyces cerevisiae. Mol. Biol. Cell 14 (2003) 129-141.

[62] Y.E. Park, et al., Autophagic degradation of nuclear components in mammalian cells. Autophagy 5 (2009) 795-804. 
[63] J. Shoji, T. Kikuma, M. Arioka, K. Kitamoto, Macroautophagy-mediated degradation of whole nuclei in the filamentous fungus Aspergillus oryzae. Plos One 5 (2010) e15650.

[64] D.C. Bassham, et al., Autophagy in development and stress responses of plants. Autophagy 2 (2006) 2-11.

[65] S. Sahara, et al., Acinus is a caspase-3-activated protein required for apoptotic chromatin condensation. Nature 408 (1999) 168-173.

[66] A.S. Haberman, M.A. Akbar, S. Ray, H. Krämer, Drosophila acinus encodes a novel regulator of endocytic and autophagic trafficking. Development 137 (2010) 2157-2166.

[67] E.J. Woltering, Death proteases: alive and kicking. Trends Plant Sci. 15 (2010) 185-188.

[68] T.A. Dittmer, N.J. Stacey, K. Sugimoto-Shirasu, E.J. Richards, LITTLE NUCLEI genes affecting nuclear morphology in Arabidopsis thaliana. Plant Cell 19 (2007) 2793-2803. 


\section{Legends of figures}

Fig. 1. Key events of nuclear dismantling during apoptosis. In animal cells the process of apoptosis culminates in nucleus dismantling. This process requires the translocation of proteins from the cytoplasm to the nucleus, which probably occurs in several waves. Some of the key events of nuclear dismantling are the proteolysis of nuclear matrix and nuclear envelope proteins, allowing the detachment of the nuclear envelope from the chromatin, the subsequent chromatin condensation and the clustering of nuclear pore complex. Cleavage of nuclear pore complex proteins provokes the disruption of nucleocytoplasmic barrier, facilitating the entrance of apoptotic factors. The proteolysis of chromatin-associated proteins close to the detachment of the nuclear envelope may open sites of nuclease hypersensitivity promoting DNA fragmentation by different nucleases. Protein phosphorylation, along with other post-translational modifications, is needed to facilitate nuclear matrix disruption, chromatin condensation and DNA fragmentation. Finally, nuclear fragmentation is produced with the formation of apoptotic bodies and engulfment by macrophages.

Fig. 2. Chromatin condensation and DNA fragmentation in human cells can be promoted by plant apoptosis-inducing factors. A, HCT116 human cells were treated with nuclear extracts from wheat nucellar cells undergoing PCD. Clear patches of chromatin condensation can be observed in the nucleus after staining with propidium iodide. B, DNA extracted from these cells was analysed in agarose gels and stained with ethidium bromide. $\mathrm{C}$, control untreated cells; $+\mathrm{N}$, samples treated with nuclear extracts from wheat nucellar cells undergoing PCD, in the absence (-) or the presence of purified Inhibitor of CAD (ICAD), $\mathrm{Zn}^{2+}$ or EDTA. This result has not previously been reported, details of experimental conditions as previously described [19].

Fig. 3. The proposed sequence of events of nuclear dismantling during plant PCD involves chromatin condensation, DNA fragmentation and nuclear envelope disruption. A, Features of chromatin condensation distinguishing ring, necklace and collapse/disassembly morphologies [39]. B, Progressive fragmentation of DNA: high molecular weight DNA cleavage, low molecular weight DNA cleavage and naked DNA unspecific cleavage. C, Nuclear envelope disassembly. Sequential cleavage of plant nucleoporins Nup 93, Nup 96, Nup 214, Nup 50, Nup 62 and Tpr/NUA was proposed 
based on apoptotic animal cells [30]. Proteolysis of plant lamin-like proteins and Nup 136 nucleoporin was proposed based on the similar role of animal lamins and Nup 155, although there is no sequence homology. 
Table 1

Relevant events in nuclear dismantling

\begin{tabular}{|c|c|c|}
\hline \multirow[t]{2}{*}{ Event } & \multicolumn{2}{|c|}{ Examples and References } \\
\hline & Animals & Plants \\
\hline Translocation to the nucleus & CAD-Inhibitor complex $[11,23,24]$ & $\begin{array}{l}\text { Transcription factor OsNAC4 [25] } \\
\text { Metacaspase mcII-Pa [26] }\end{array}$ \\
\hline $\begin{array}{l}\text { Processing of apoptogenics factors } \\
\text { or cleavage of their inhibitors }\end{array}$ & $\begin{array}{l}\text { Cleavage of CAD inhibitor by caspase- } 3 \\
{[24]}\end{array}$ & $\begin{array}{l}\text { Cleavage of tudor staphylococcal nuclease } \\
\text { (TSN) by a metacaspase mcII-Pa [27] }\end{array}$ \\
\hline $\begin{array}{l}\text { Disruption of nucleocytoplasmic } \\
\text { barrier }\end{array}$ & $\begin{array}{l}\text { Degradation of cytoplasmic filaments and } \\
\text { nuclear basket from nuclear pore complex } \\
\text { [29] }\end{array}$ & n.d. \\
\hline $\begin{array}{l}\text { Protection of cleavage sites of some } \\
\text { nucleoporins }\end{array}$ & Nucleoporin Nup62 [30] & n.d. \\
\hline \multicolumn{3}{|l|}{$\begin{array}{l}\text { Accessibility to the conformation of } \\
\text { DNA }\end{array}$} \\
\hline - Chromatin loops & Chromatin loop nuclease [31] & Chromatin loop nuclease [32] \\
\hline - Internucleosomal DNA & CAD nuclease $[22,23]$ & $\begin{array}{l}\text { D-mannose-induced nuclease [33] } \\
\text { PCD-related nuclease }[15,34]\end{array}$ \\
\hline - Naked DNA & Different nucleases [11] & ZEN1 nuclease [35] \\
\hline Nuclear Pore Complex clustering & $\begin{array}{l}\text { Nuclear Pore Complex clustering in } \\
\text { apoptosis [19] }\end{array}$ & $\begin{array}{l}\text { Nuclear Pore Complex clustering in plant } \\
\text { PCD [32] }\end{array}$ \\
\hline Nuclear fragment engulfment & $\begin{array}{l}\text { Phagocytosis of apoptotic bodies by } \\
\text { macrophages [36] }\end{array}$ & $\begin{array}{l}\text { Autophagic process to remove nuclear } \\
\text { fragments }[13,32]\end{array}$ \\
\hline
\end{tabular}

n.d.: not determined 
Table 2

Sequence of cytoplasmic events preparing for plant nuclear dismantling

\begin{tabular}{|c|c|c|}
\hline Organelle & Event & References \\
\hline ER-derived compartments & $\begin{array}{l}\text { Accumulation and release of cysteine proteases and nucleases in } \\
\text { the onset of PCD }\end{array}$ & {$[41,55]$} \\
\hline ER-Golgi & $\begin{array}{l}\text { Chaperoning and inhibition of cysteine proteases by protein } \\
\text { disulfide isomerase- } 5 \text { during trafficking to vacuole before PCD }\end{array}$ & [56] \\
\hline $\begin{array}{l}\text { Vacuole (PSV and Lytic } \\
\text { Vacuole) }\end{array}$ & $\begin{array}{l}\text {-Self-processing of vacuolar processing enzyme at acid } \mathrm{pH} \\
\text {-Maturation of precursors of PR proteins and hydrolases by } \\
\text { vacuolar processing enzyme } \\
\text {-Accumulation of aspartate proteases, cysteine proteases and } \\
\text { nucleases until their release in PCD } \\
\text {-Tonoplast disruption }\end{array}$ & {$[4,57]$} \\
\hline Mitochondria & $\begin{array}{l}\text { Release of endonucleases, cytochrome } \mathrm{c} \text { and other apoptogenic } \\
\text { factors }\end{array}$ & [58] \\
\hline Choroplast & Release of ROS & [59] \\
\hline Cytoskeleton & $\begin{array}{l}\text { Depolymerization of microtubule and reorganization of actin } \\
\text { network providing a skeleton to trigger autophagosome } \\
\text { formation and promoting autophagic movements for cell content } \\
\text { engulfment }\end{array}$ & {$[60]$} \\
\hline $\begin{array}{l}\text { Autophagosomes and } \\
\text { autophagic vacuoles }\end{array}$ & Digestion of remnants of membrane-less nuclear fragments & {$[13,32,40]$} \\
\hline
\end{tabular}




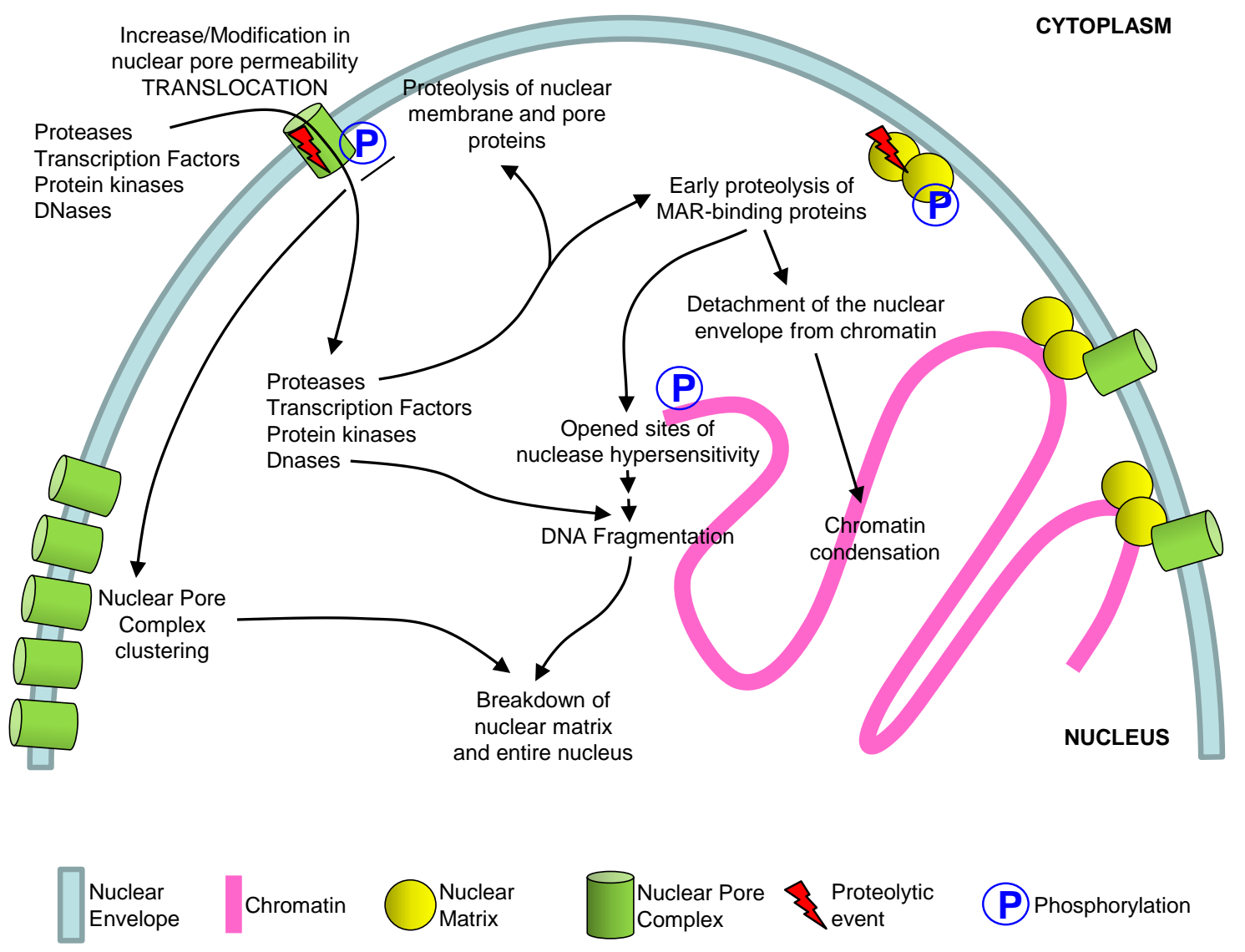

Figure 1 

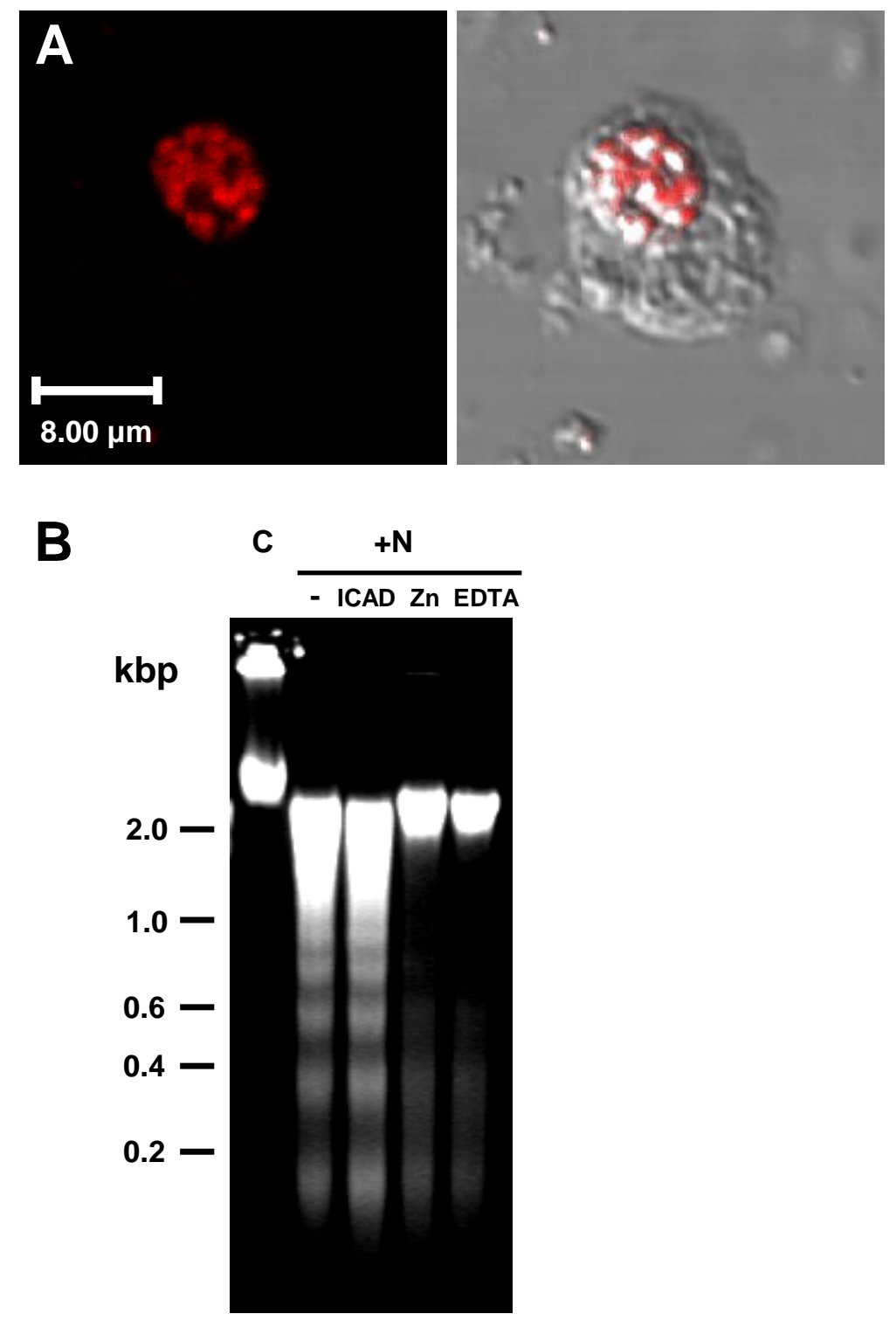

Figure 2 
Stage I

\section{A Chromatin Condensation}

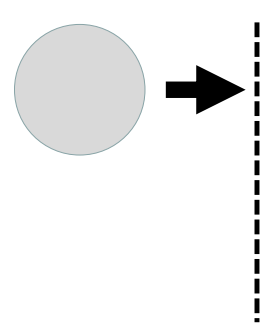

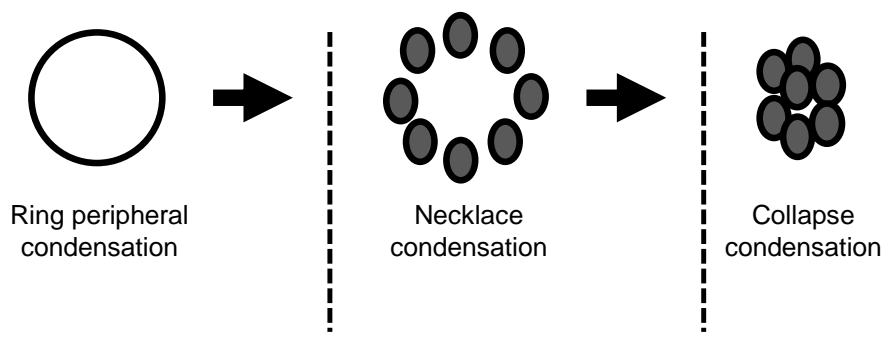

\section{B DNA Fragmentation}

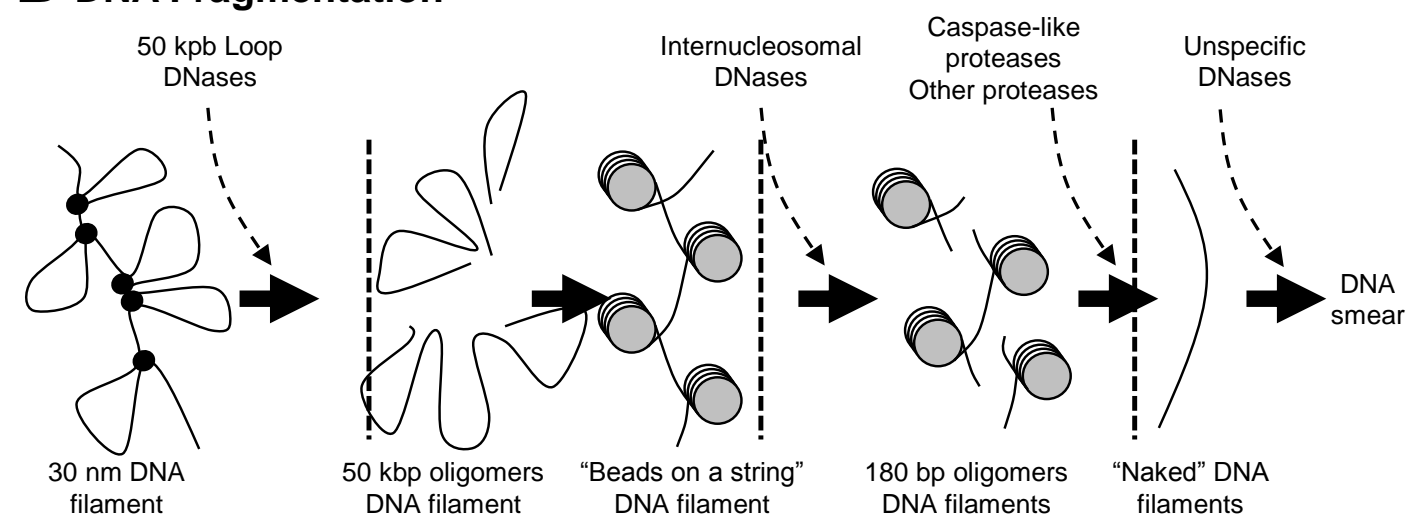

\section{Nuclear Envelope / Nuclear Pore Disassembly}
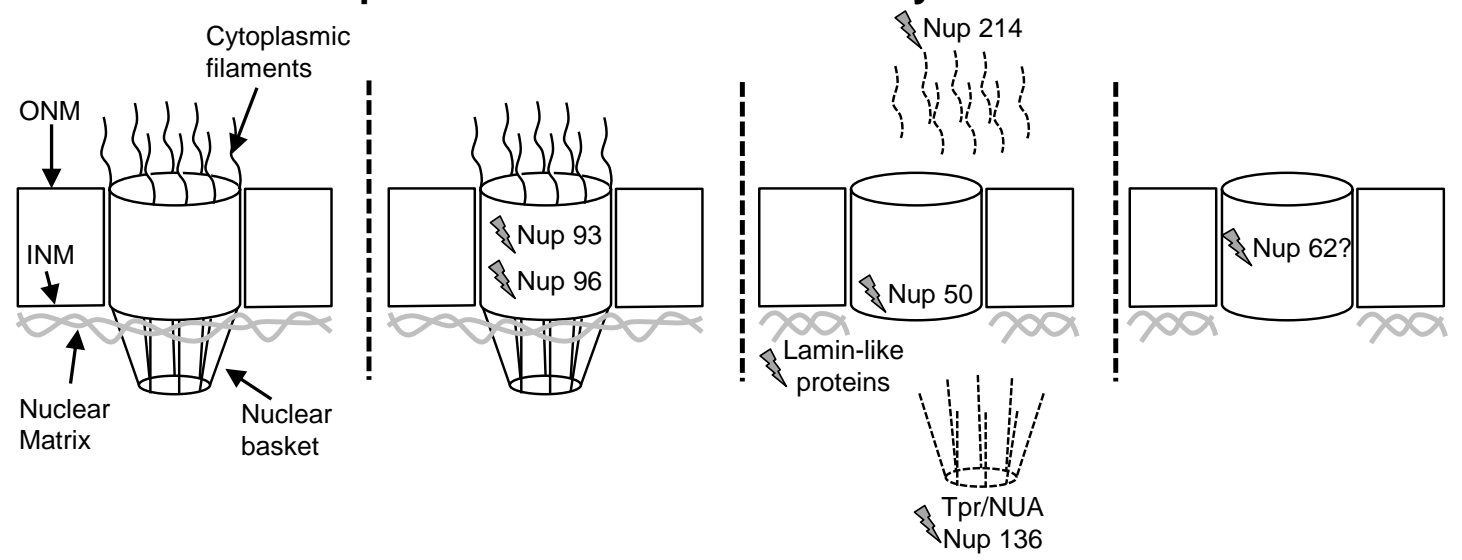

Figure 3 